poorer cognition at 7 years. This indicates that early aEEGs may be useful in predicting functional outcome of preterm children at school age.

\section{NEURODEVELOPMENTAL OUTCOME AMONG MULTIPLES AND SINGLETONS: A REGIONAL NEONATAL INTENSIVE CARE UNIT'S EXPERIENCE IN TURKEY}

doi:10.1136/archdischild-2012-302724.1231

$1 Z$ Eras, ${ }^{2 B M}$ Özyurt, ${ }^{3} 0$ Erdeve, 'ED Sakrucu, ${ }^{2} S S$ Oguz, ${ }^{2} F E$ Canpolat, ${ }^{2} \mathrm{~N}$ Uras, ${ }^{4} \mathrm{U}$ Dilmen. ${ }^{1}$ Developmental Behavioral Pediatrics Unit; ${ }^{2}$ Neonatology, Zekai Tahir Burak Maternity and Teaching Hospital; ${ }^{3}$ Neonatology, Ankara University, Faculty of Medicine; ${ }^{4}$ Neonatology, Zekai Tahir Burak Maternity and Teaching Hospital, Yıldırım Beyazıt University Department of Pediatrics, Ankara, Turkey

Objective The aim of this study was to compare the neurodevelopmental outcome at 12-18 months' corrected age between multiples and singleton preterm infants.

Methods We designed a prospective study of preterm infants $(\leq 32$ weeks gestation) born and hospitalized in the neonatal intensive care unit between November 2008 and November 2009, whose assessments were performed at 12-18 months' corrected age. Neurodevelopmental impairment was defined as the presence of any one of the following: moderate or severe cerebral palsy, severe bilateral hearing loss or bilateral blindness, mental developmental index score or psychomotor developmental index score less than 70 . Results were compared for both multiples and singleton infants.

Results A hundred and fifty-nine multiples and 211 singleton infants were assessed at 12-18 months' corrected age. The neurodevelopmental outcome including all parameters at 12-18 months' corrected age in multiples was not significantly different from singleton preterm infants.

Conclusions Multiple gestation in preterm infants is not associated with an increased risk of neurodevelopmental impairment at 12-18 months' corrected age compared to singleton preterm infants. For further information long-term and high-participated neurodevelopmental follow-up and evaluation at pre-school age will be needed.

\section{DO ORAL PROBIOTICS AFFECT GROWTH AND NEURODEVELOPMENTAL OUTCOMES IN VERY LOW BIRTH WEIGHT PRETERM INFANTS?}

doi:10.1136/archdischild-2012-302724.1232

${ }^{1} \mathrm{FN}$ Sarı, ${ }^{2} \mathrm{Z}$ Eras, ${ }^{3} \mathrm{EA}$ Dizdar, ${ }^{4} 0$ Erdeve, ${ }^{3} \mathrm{SS}$ Oguz, ${ }^{3} \mathrm{~N}$ Uras, ${ }^{5} \mathrm{U}$ Dilmen. ${ }^{1}$ Noenatology; ${ }^{2}$ Developmental Behavioral Pediatrics Unit; ${ }^{3}$ Neonatology, Zekai Tahir Burak Maternity and Teaching Hospital; "Neonatology, Ankara University, Faculty of Medicine${ }^{5}$ Neonatology, Zekai Tahir Burak Maternity and Teaching Hospital/YIIdırım Beyazıt University Department of Pediatrics, Ankara, Turkey

Objective To evaluate the growth and neurodevelopmental outcomes of very low birth weight (VLBW) preterm infants supplemented with oral probiotics for the prevention of necrotising enterocolitis (NEC).

Study Design This prospective follow-up study was conducted in a cohort of VLBW preterm infants enrolled in a randomizedcontrolled clinical trial to evaluate the efficacy of oral probiotics for the prevention of NEC. Growth outcomes included weight, length and head circumference. Neurodevelopmental assessment was done using the Bayley Scales of Infant Development II (BSID-II). The primary outcome was neurodevelopmental impairment at 18-24 months' corrected age.

Results A total of 221 infants completed the trial protocol. Of the 208 infants eligible for follow-up, 174 infants (86 in the probiotics group and 88 in the control group) were evaluated. There were no significant difference in growth and neurodevelopmental outcomes between the two groups.
Conclusion Oral probiotic administrated to VLBW infants to reduce the incidence and severity of NEC started with the first feed did not affect growth and neurodevelopmental outcomes at 18-24 months' corrected age.

\section{THE IMPACT OF MEDICAL HOME MODEL ON NEURODEVELOPMENTAL OUTCOMES OF VERY PRETERM INFANTS}

doi:10.1136/archdischild-2012-302724.1233

'Z Eras, ${ }^{2} \mathrm{G}$ Kanmaz, ${ }^{2} \mathrm{BM}$ Ozyurt, ${ }^{2} \mathrm{FE}$ Canpolat, ${ }^{1} \mathrm{ED}$ Sakrucu, ${ }^{3} \mathrm{U}$ Dilmen. ${ }^{1}$ Developmental Behavioral Pediatrics Unit; ${ }^{2}$ Neonatology, Zekai Tahir Burak Maternity and Teaching Hospital; ${ }^{3}$ Neonatology, Zekai Tahir Burak Maternity and Teaching Hospital, Yıldırım Beyazit University Department of Pediatrics, Ankara, Turkey

Objective The aim of the study is to compare the 2-year neurodevelopmental outcome of very low birth weight and/or very preterm infants followed with "medical home model" implemented in a big maternity hospital in Turkey with the primary health care.

Methods We designed a prospective controlled study of infants born $\leq 32$ week's gestation and/or $\leq 1500 \mathrm{~g}$ and hospitalized. After discharge, intervention group were followed with in the concept of medical home model and control group were followed in the concept of primary standart health care. At 18-24 months' of corrected age, the neurological examinations and the developmental assessments using the Bayley Scales of Infant Development-II were performed.

Results The cerebral palsy rates in the intervention and control group were $10.6 \%(n=17)$ and $17.4 \%(n=27)$ respectively and the difference was not statistically significant $(p=0.107)$. Whereas the rate of neurodevelopmental impairment in the intervention group was significantly lower than the control group $(25 \%$ and $38,7 \%$; $\mathrm{p}=0.011$ ).

Conclusion We demonstrated that beginning a good follow-up process within the context of medical home model, efficient developmental support and performing guidance for early diagnose reduced the negative effects of VLBW and/or prematurity on neurodevelopmental impairment at 2 years of age.

\section{4 PREDICTORS OF LITERACY IN VERY PRETERM INFANTS: A LONGITUDINAL STUDY}

doi:10.1136/archdischild-2012-302724.1234

'A Guarini, 'A Sansavini, 'S Savini, ${ }^{2} \mathrm{R}$ Alessandroni, ${ }^{2} \mathrm{G}$ Faldella. 'Department of Psychology; ${ }^{2}$ Institute of Neonatology, University of Bologna, Bologna, Italy

Background and Aims As revealed by several studies, a very preterm birth may affect language, executive functions, verbal short term memory and literacy. The aim of this study was to investigate these competencies in very preterms compared to full terms, thus describing how language, executive functions and verbal short-term memory observed at the end of preschool age affect literacy at school age.

Methods Forty-two monolingual Italian very preterms (mean gestational age 29.7 weeks), with no history of major cerebral damage, were followed longitudinally at 6 years by investigating language (vocabulary, grammar, and phonological awareness), executive functions (syllabic and phonemic fluency) and verbal short-term memory (vowel span, non-word repetition) and again at 8 years on literacy (word and non-word reading and spelling). Two crosssectional groups of full term children were recruited as control groups ( 34 and 26 children at 6 and 8 years respectively).

Results Preterms showed lower abilities in language at 6 years and in literacy at 8 years, while no differences between groups regarding syllabic and phonemic fluency and short-term memory were found. Linear regression analyses revealed that in the preterm sample both language and verbal short-term memory had a predictive role on 